Copyright 2008 Society of Photo-Optical Instrumentation Engineers. This paper was published in Proc. SPIE 7020 and is made available as an electronic reprint with permission of SPIE. One print or electronic copy may be made for personal use only. Systematic or multiple reproduction, distribution to multiple locations via electronic or other means, duplication of any material in this paper for a fee or for commercial purposes, or modification of the content of the paper are prohibited.

\title{
Performance of the SCUBA-2 dilution refrigerator
}

\author{
Matthew Hollister ${ }^{a}$, Adam Woodcraft ${ }^{a, b}$, Wayne Holland ${ }^{b}$ and Dan Bintley ${ }^{c}$ \\ ${ }^{a}$ SUPA, Institute for Astronomy, Royal Observatory, Edinburgh, EH9 3HJ, UK; \\ ${ }^{b}$ UK Astronomy Technology Centre, Royal Observatory, Edinburgh, EH9 3HJ, UK; \\ ${ }^{c}$ Joint Astronomy Centre, 660 N. A'ohoku Place, Hilo, HI 96720, USA
}

\begin{abstract}
SCUBA-2 is a new wide-field submillimeter continuum instrument being commissioned on the James Clerk Maxwell Telescope on Mauna Kea in Hawaii. SCUBA-2 images simultaneously at 450 and $850 \mu \mathrm{m}$ using largescale arrays of superconducting bolometers, with over five thousand pixels at each wavelength. The arrays are cooled to less than $100 \mathrm{mK}$ by the mixing chamber of a dilution refrigerator (DR), with a radiation shield at a nominal temperature of $1 \mathrm{~K}$ cooled by the DR still. The DR is a "dry" system, using a pulse tube cooler for precooling of the circulating helium in place of a liquid helium bath. This paper presents key performance data for the DR.
\end{abstract}

Keywords: SCUBA-2, Submillimeter bolometer array, Cryogenics, Dilution refrigerator, Pulse tube cooler

\section{INTRODUCTION}

The submillimeter common-user bolometer array-2 (SCUBA-2) is a wide-field survey camera presently being commissioned on the James Clerk Maxwell Telescope on Mauna Kea in Hawaii. SCUBA-2 images at 450 and $850 \mu \mathrm{m}$ using large-scale arrays of superconducting bolometers, operating at less than $100 \mathrm{mK}$. More detailed descriptions of the instrument and science goals may be found elsewhere. ${ }^{1}$

The detector arrays are cooled by the mixing chamber of a liquid cryogen-free (or "dry") dilution refrigerator (DR) developed by Leiden Cryogenics, ${ }^{*}$ using a two-stage pulse tube cooler (PTC) in place of liquid nitrogen and helium baths as in conventional systems. A number of groups have developed such systems for specific applications. $^{2-4}$ Low temperature cryostats using mechanical coolers are typified by ease of operation, since there is no need for the expense and technical difficultly of using liquid ${ }^{4} \mathrm{He}$ to achieve temperatures of $\sim 4 \mathrm{~K}$. Dry DRs are now available commercially from a number of companies as demand for such "turn key" millikelvin cryostats has increased in recent years. The SCUBA-2 DR represents an early prototype of such commercial systems.

The specified performance for the SCUBA-2 DR required an unloaded mixing chamber base temperature of less than $10 \mathrm{mK}$, and providing a cooling power of $500 \mu \mathrm{W}$ at $120 \mathrm{mK}$, and $30 \mu \mathrm{W}$ at $65 \mathrm{mK}$ (with a goal of $30 \mu \mathrm{W}$ at $35 \mathrm{mK}$ ). These figures are taken from the stated performance characteristics of the conventional Leiden Cryogenics dilution refrigerator upon which the DR was to be based. The still was to run at a temperature of $<900 \mathrm{mK}$, with a goal of $<700 \mathrm{mK}$, while providing at least $5 \mathrm{~mW}$ of capacity to cool cryogenic amplifiers, filters and radiation shielding. More detailed descriptions of the cryogenic design of SCUBA-2 are available elsewhere. ${ }^{5,6}$

The paper describes the baseline performance of the dilution refrigerator in standalone operation (outside the instrument cryostat), along with some discussion of the system performance when integrated with the full instrument. A description of the key design features of the DR is given in $\S 2$, and the behaviour of the refrigerator during cooldown is discussed in $\S 3$. The main performance characteristics are described in $\S 4$.

Further author information: (Send correspondence to M.H.) e-mail: matt.hollister@physics.org

* Leiden Cryogenics BV, Galgewater no.21, 2311 VZ Leiden, The Netherlands (http://www.leidencryogenics.com)

Millimeter and Submillimeter Detectors and Instrumentation for Astronomy IV edited by William D. Duncan, Wayne S. Holland, Stafford Withington, Jonas Zmuidzinas Proc. of SPIE Vol. 7020, 70200Y, (2008) · 0277-786X/08/\$18 - doi: 10.1117/12.787791

Proc. of SPIE Vol. 7020 70200Y-1 


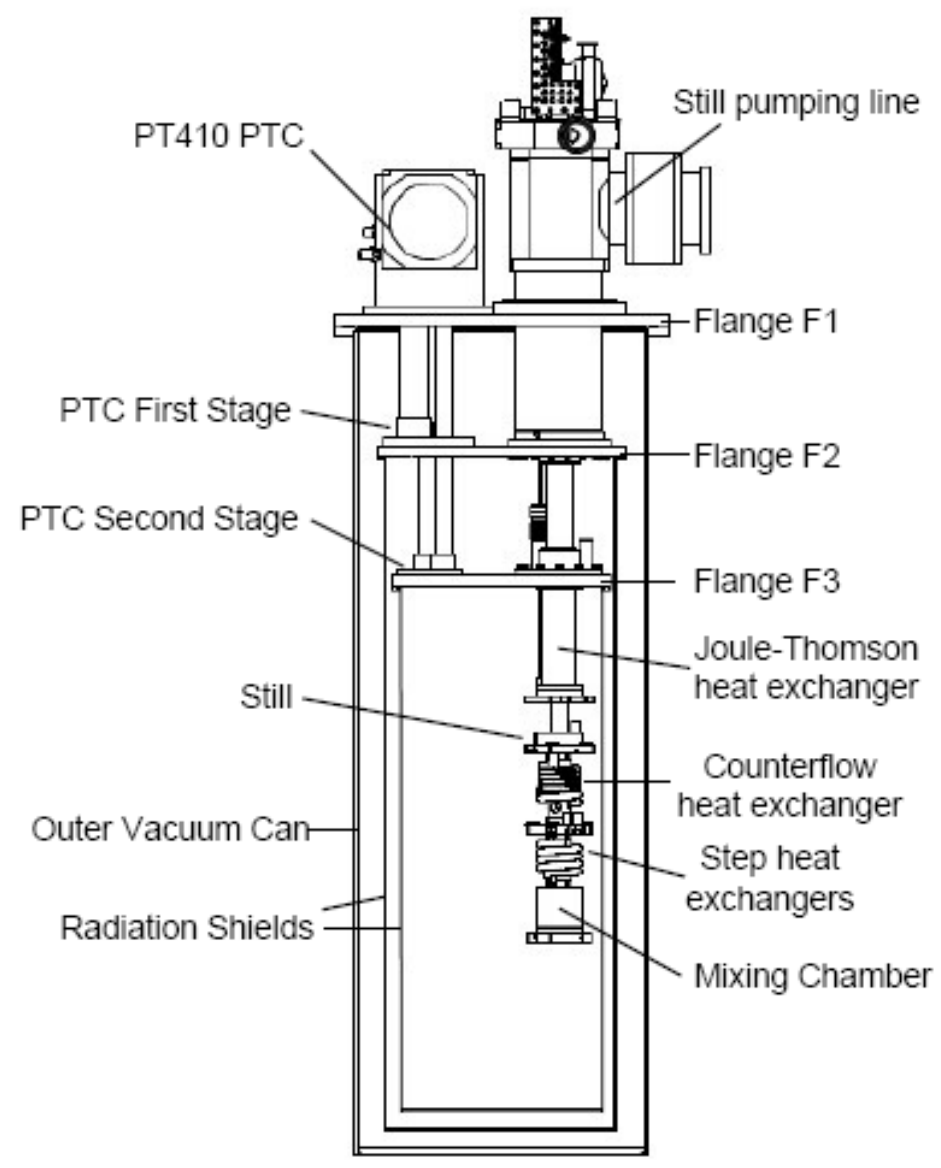

Figure 1. The SCUBA-2 dilution refrigerator insert in the test cryostat (shown in cross-section), with key features indicated. See text for details.

\section{DESCRIPTION OF THE DILUTION REFRIGERATOR}

The SCUBA-2 dilution refrigerator was designed and constructed by Leiden Cryogenics, based on a conventional Leiden MNK-126-500 dilution unit with the 1-K pot replaced by a Joule-Thomson (JT) stage. The JT stage is a counterflow heat exchanger in which the instreaming helium is cooled by the gas pumped from the still, followed by an impedance to produce liquifaction by expansion. ${ }^{7}$ A number of modifications were carried out to the dilution refrigerator and gas handling system following delivery and commissioning. This section gives an overview of the DR. Many of the specific details of the dilution refrigerator design are not included due to the information being proprietary to Leiden Cryogenics.

Due to the adoption of the methodology of having seperate sub-systems that could be tested independently, the dilution refrigerator was designed as a self-contained insert for the instrument cryostat. The "insert" refers to the full refrigerator, including the pulse tube cooler and precooling stages of the DR, as well as the dilution stage (still and mixing chamber). A schematic of the insert is shown in Fig. 1, with the major features indicated. A block diagram of the refrigerator and room temperature pumping system is shown in Fig. 2. 


\subsection{Pulse tube cooler}

The PTC used for the SCUBA-2 DR is a Cryomech ${ }^{\dagger} \mathrm{PT}_{4} 10$, a two-stage refrigerator with a nominal cooling capacity of $1 \mathrm{~W}$ at a temperature of $4.2 \mathrm{~K}$ on the second stage and an unloaded base temperature of approximately $2.5 \mathrm{~K}$. The first stage of the cooler will provide $40 \mathrm{~W}$ at $45 \mathrm{~K}$. The pulse tube is driven by a CP980 compressor package with an input power of $8 \mathrm{~kW}$.

\subsection{Insert construction}

The top of the insert (flange F1 in Fig. 1) is a stainless steel plate, supporting the top of the still pumping line and the room temperature vacuum flange of the pulse tube cooler. The top of the DR pumping line consists of a steel box supporting the output flange of the still pumping line (ISO100 fitting with pneumatic gate valve), the helium input line (NW16 flange isolated by solenoid valves), and four clear shot ports. The clear shot tubes come out in the vacuum can below the first stage plate. The clear shot tubes carry the DR wiring, and allow the drive mechanisms for mechanical heatswitches and the impedance bypass valve (see below) to enter the vacuum can.

The first stage plate (flange F2) is a copper plate that interfaces to the DR pumping line and is cooled by the first stage of the pulse tube cooler. The plate is bolted directly to the first stage heat exchanger flange of the PTC. The still pumping line is a solid stainless steel pipe. The helium input capillaries exchange heat with a copper flange that is interfaced to the first stage plate, precooling the incoming helium. The second stage plate (flange F3) is also of copper. The second stage of the PTC is bolted directly to this plate. The DR pumping line between the first and second stage plates is again stainless steel. The twin helium input lines pass through $13 \mathrm{~mm}$ diameter cold trap volumes of copper and stainless steel shavings to clean the mixture. Above the F3 flange, the input capillaries are run inside the still pumping line as in a standard Leiden DR, although for this fridge heat exchange at the second stage is achieved with a sintered block heat exchanger bolted to the underside of the copper plate. The twin input capillaries (copper-nickel, $1.4 \mathrm{~mm}$ outside diameter, $1.2 \mathrm{~mm}$ internal diameter) exit the still pumping line, pass through the sinter block, and reenter the pumping line immediately below the F3 flange.

To aid the precool of the dilution refrigerator, a heatswitch is mounted on both the first and second stage plates. The heatswitches are mechanical, driven manually by drive shafts from the top of the insert. The first stage switch is mounted on the underside of the first stage plate (F2 in Fig. 1, closing on a gold plated copper bar bolted to the second stage plate, effectively shorting the two stages of the PTC together, utilising the cooling power of the first stage to cool the second stage. The second stage switch is mounted on the top face of the second stage plate (F3 in Fig. 1), closing on a segmented bar feeding through the second stage plate and terminating at the still. The cold bar is isolated from the second stage plate by a support of Kevlar thread under tension. The primary purpose of the links is to aid the precool of the 1-K structures in the instrument by providing a thermal path to the DR pulse tube cooler.

\subsection{Dilution unit}

The dilution unit is based on a Leiden Cryogenics MNK-126-500 DR, with a Joule-Thomson (JT) heat exchanger in place of the 1-K pot. After precooling at the second stage, the helium enters the JT, a counterflow heat exchanger with the input capillaries coiled inside the stainless steel pumping line. The design of the JT stage is similar to that described by Uhlig. ${ }^{7}$ The input lines pass independently through flow impedances at the end of the counterflow exchanger. ${ }^{\ddagger}$ One impedance line is fitted with a needle valve bypass to allow a high flow rate during helium condensation. The needle valve is manually driven by a micrometer mechanism on the top of the insert.

The input capillaries join into a single line below the JT, and then enter the still. On leaving the still, the helium passes through a counterflow heat exchanger. Below the counterflow exchanger, the liquid passes through a single silver sinter step exchanger. An intermediate plate is attached below the step heat exchanger to provide heat sinking. The helium capillary passes through a further six step exchangers, before entering the

\footnotetext{
${ }^{\dagger}$ Cryomech, Inc., 113 Falso Drive, Syracuse, NY 13211, USA (http://www.cryomech.com)

${ }^{\ddagger}$ Essentially, there are two Joule-Thomson stages in parallel
} 


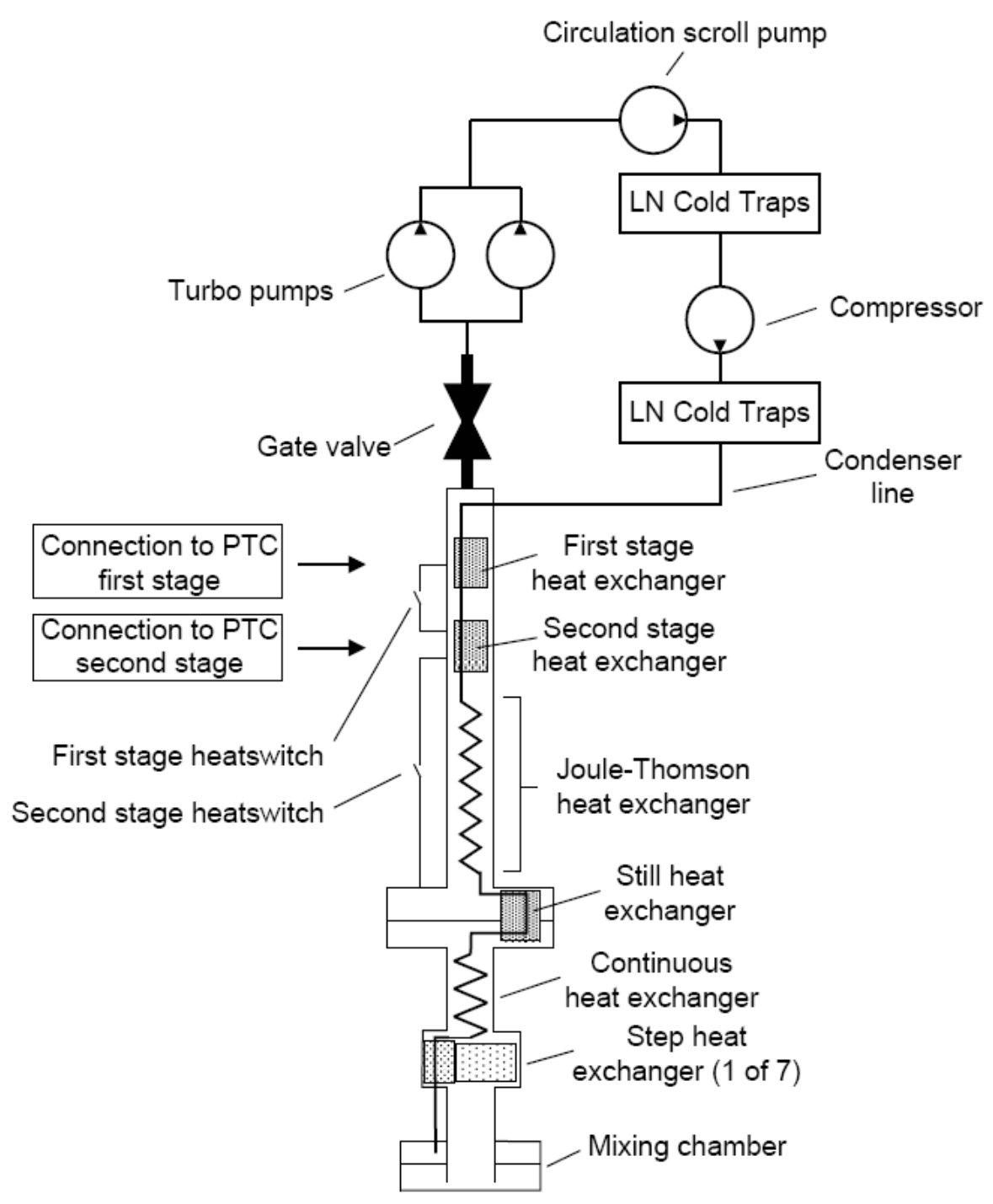

Figure 2. Block diagram of the SCUBA-2 dilution refrigerator and room temperature pumping system.

mixing chamber. The mixing chamber is of aluminium alloy, with a gold-plated aluminium alloy plate welded to the bottom of the mixing chamber for sample mounting. The intermediate plate and the mixing chamber are supported by a G10 composite tube.

\subsection{Thermometry}

The dilution refrigerator was delivered with a set of six Leiden Cryogenics resistive thermometers on the various stages of the insert. $1 \mathrm{k} \Omega$ platinum devices are attached to the first stage plate and to the precool link between the first and second stages. $10 \mathrm{k} \Omega$ ruthenium oxide devices are attached to the second stage plate, the output flange of the JT heat exchanger, and in the still (immersed in the dilute phase). The mixing chamber thermometer is a $360 \Omega$ Speer carbon device. These thermometers were supplied with generic calibration curves. The mixing chamber thermometer was subsequently calibrated against a Lake Shore ruthenium oxide sensor. The calibration curves of the Leiden ruthenium oxide thermometers were found to be reasonable above $2 \mathrm{~K}$ when checked against 
independently calibrated devices. The sensors are read out using a Lake Shore 370 AC resistance bridge ${ }^{\S}$.

The thermometry was later supplemented with a number of Lake Shore RX-102 ruthenium oxide sensors at the input and output of the JT heat exchanger, and on the still coldplate. These sensors were calibrated between 0.5 and $4 \mathrm{~K}$ against a Lake Shore germanium thermometer. An uncalibrated RX-102 was fitted to the second stage of the PTC as an additional diagnostic.

\subsection{Gas handling system}

The gas handling system (GHS) is a self-contained cabinet constructed from hollow square-section stainless steel tubing, welded to produce two leak-tight reservoirs for storage of the helium mixture. The upper tank, volume 36 litres, is used for the ${ }^{3} \mathrm{He}$-rich mixture, while the remaining volume (146 litres) holds the ${ }^{4} \mathrm{He}$-rich mixture.

Helium is circulated around the dilution circuit by twin turbo pumps (Varian Navigator 551) in parallel, backed by a sealed scroll pump (BOC Edwards XDS35i). A diaphragm compressor (KNF Neuberger N143AN.12E) is in series after the scroll pump to increase the rate at which helium is condensed into the DR, and to reduce the output pressure of the scroll pump at high circulation rates. The DR is pumped through a $100 \mathrm{~mm}$ internal diameter pumping line, $2 \mathrm{~m}$ in length, connected to the gate valve on the insert. Operational requirements at the telescope required a large separation between the instrument cryostat and GHS, so this pumping line was later replaced with a $10 \mathrm{~m}$ line.

Liquid nitrogen cold traps are used to clean the returning mixture, with two traps in parallel after the scroll pump and twin traps after the compressor. Helium is returned to the input side of the dilution circuit via a $4 \mathrm{~m}$ long NW10 flexible line with $10 \mathrm{~mm}$ internal diameter. As with the still pumping line, the input line was also subsequently replaced by a $10 \mathrm{~m}$ line. The cold traps are fitted with additional valves to isolate and pump out individual traps without interrupting the circulation of mixture.

\subsection{Cryostat interfaces}

The work described in this paper deals both with the operation of the DR in a standalone test cryostat, and interfaced to the main instrument cryostat. The test cryostat is a stainless steel can that interfaces to the insert vacuum flange F1, with copper radiation shields mounted to the F2 and F3 flanges. In the instrument cryostat, the F2 and F3 flanges interface to the instrument radiation shields via stainless steel bellows. In addition, a radiation shield is mounted on the still, surrounding the lower temperature stages of the DR from $4 \mathrm{~K}$ radiation. Wicks run from the still and mixing chamber to cool the 1-K radiation shield and focal planes, respectively.

\section{OPERATION OF THE DILUTION REFRIGERATOR}

A cooldown curve for the precool of the insert in the test cryostat is shown in Fig. 3. To aid the precool of the insert, the ${ }^{4} \mathrm{He}$-dump is opened to the input side of the dilution refrigerator through the cold traps before the pulse tube cooler is started. As the insert cools, the ${ }^{4} \mathrm{He}$ will condense into the dilution unit. Although this process does not reduce the precool time in the test cryostat, it does reduce the time to condense the helium mash into the DR after precool since most of the ${ }^{4} \mathrm{He}$ is already liquified, which lowers the temperature of the still and the JT heat exchanger (which tend to lag behind the PTC and mixing chamber temperatures).

The first and second stage plates, the still and JT all cool below $100 \mathrm{~K}$ in less than 10 hours, with the mixing chamber lagging due to the poor thermal path to the bottom of the insert. The first stage heatswitch is opened after 8 hours, once the second stage plate reaches the temperature of the first stage (approximately $50 \mathrm{~K})$. Beyond this point, the short between the two stages offers no benefit. The second stage switch remains closed for the full period of the precool and condensing phases. The first stage plate cools to a temperature of $37 \mathrm{~K}$, while the second stage plate cools below $3 \mathrm{~K}$ with no helium load. Due to the lag in the mixing chamber temperature, the full precool process (dilution unit temperatures below $4 \mathrm{~K}$ ) takes 23 hours to complete.

Once the precool is complete, the majority of the ${ }^{4} \mathrm{He}$ charge has been liquified in the dilution unit. The remaining charge is transfered to the insert using the circulation scroll pump and the compressor. The ${ }^{3} \mathrm{He}$ charge is then added to the insert in the same way. The helium charge for the DR is composed of 140 litres

\footnotetext{
${ }^{\S}$ Lake Shore Cryotronics, Inc., 575 McCorkle Blvd, Westerville, OH 43082, USA (http://www.lakeshore.com)
} 

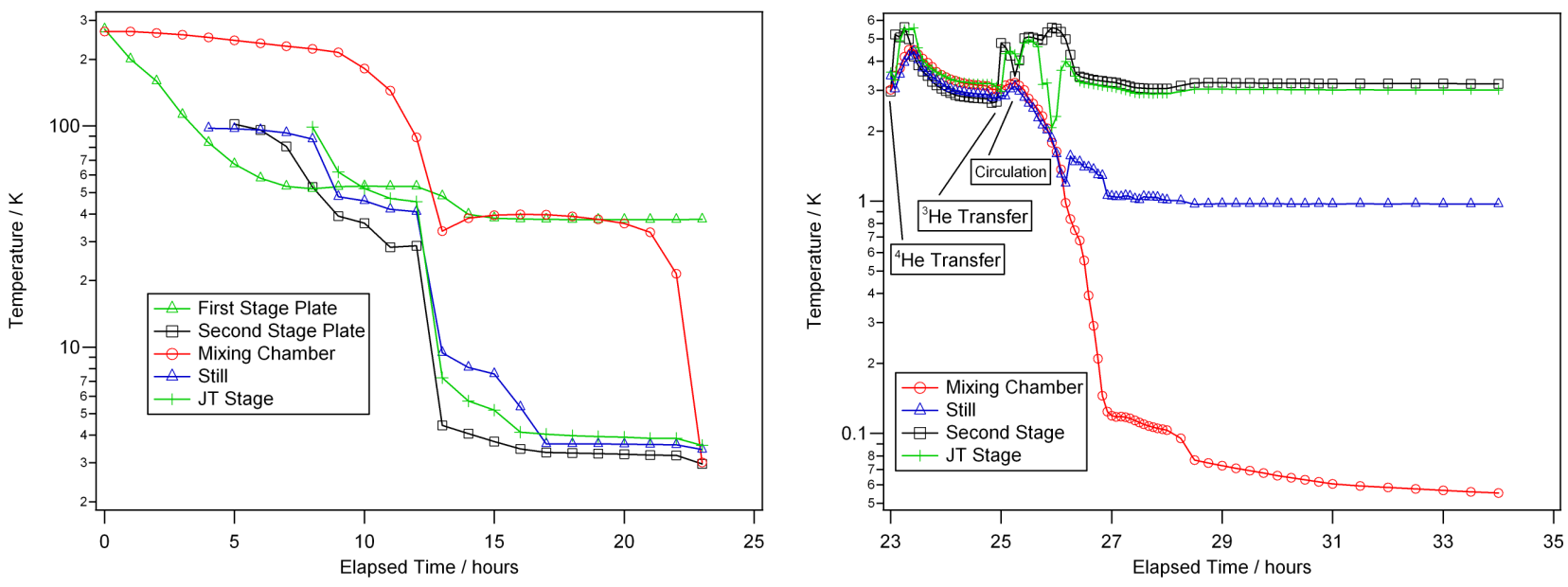

Figure 3. Cooldown of the dilution refrigerator. Left: precooling to $\sim 4 \mathrm{~K}$. Right: condensation of mixture and cooldown to base temperature.

of ${ }^{4} \mathrm{He}$ and 32 litres of ${ }^{3} \mathrm{He}$. The transfer of gas to the insert causes the second stage and JT temperatures to rise to approximately $5 \mathrm{~K}$, with smaller rises on the still and mixing chamber. This temperature spike lasts approximately 20 minutes before the stages cool and the gas liquifies. The second stage heatswitch is closed during the gas transfers to reduce the temperature spike on the still associated with the warm gas flow. Without the short to the second stage plate, it was found that the still could rise to $10 \mathrm{~K}$ or more as gas was added from the dumps, increasing the input pressure and prolonging the process of condensing the gas mixture.

The condensing process and cooldown to final base temperature is shown in Fig. 3. Once the full charge is condensed in the insert, the second stage heatswitch is opened and the mixture is circulated using the scroll pump and compressor. The flow is regulated manually using a valve on the turbo pump backing line, keeping the input pressure below 4 bar. Without regulation, the input pressure can spike to 10-15 bar, greatly increasing the risk of mixture loss from room temperature o-rings.

Once the regulation valve is fully open and the mash flowing freely, the turbo pumps are started, spinning up to full speed over $\sim 20$ minutes, reducing the still pressure and cooling the dilution unit. The temperature of the JT stage remains at approximately $3 \mathrm{~K}$ during the process, although at high input pressures (2-4 bar) early in the condensing process, the JT output temperature can reach as low as $1.5 \mathrm{~K}$. During normal circulation, the temperature of the second stage plate (approximately equal to the input temperature of the JT heat exchanger) is $3.2 \mathrm{~K}$.

When the mixing chamber cools below $1 \mathrm{~K}$, the bypass valve on the primary input line is closed. This increases the flow impedance, and increases the cooling effect. The second input line is closed once the mixing chamber has cooled below $800 \mathrm{mK}$. The temperature falls rapidly to $\sim 100 \mathrm{mK}$, after which the cooling rate flattens out towards the final base temperature of $\sim 45 \mathrm{mK}$ with $14 \mathrm{~mW}$ of heat applied to the still. The total cooldown time from room temperature to base temperature is 34 hours.

\section{DILUTION REFRIGERATOR PERFORMANCE}

The performance of the dilution refrigerator was extensively determined during operation in the test cryostat in order to provide a baseline for performance comparisons to operation in the main instrument cryostat. The cryogenic design of the instrument is such that the DR insert is well isolated from the $60-\mathrm{K}$ and $4-\mathrm{K}$ stages of the instrument cryostat. Essentially, the DR will behave in nominally the same way in both cryostats, with the exception of the static loads on the still and mixing chamber. This section will describe the baseline performance of the system in the test cryostat, and then cover some of the key performance parameters in the instrument cryostat. 


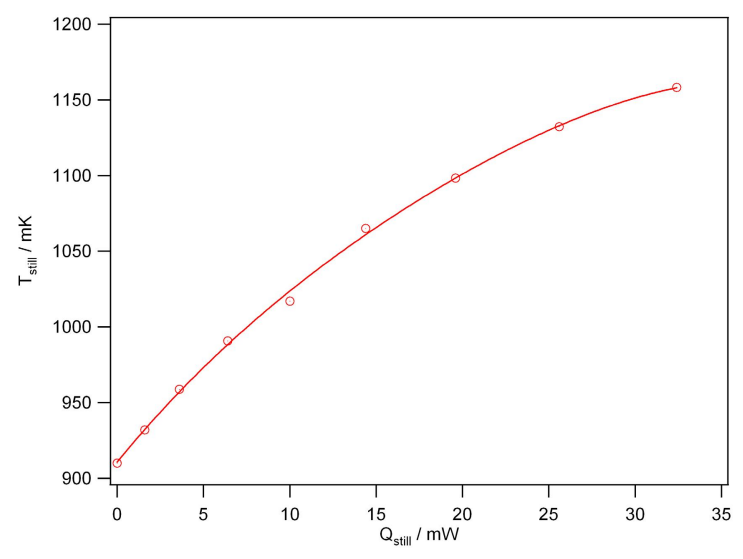

Figure 4. Cooling capacity of the dilution refrigerator still.

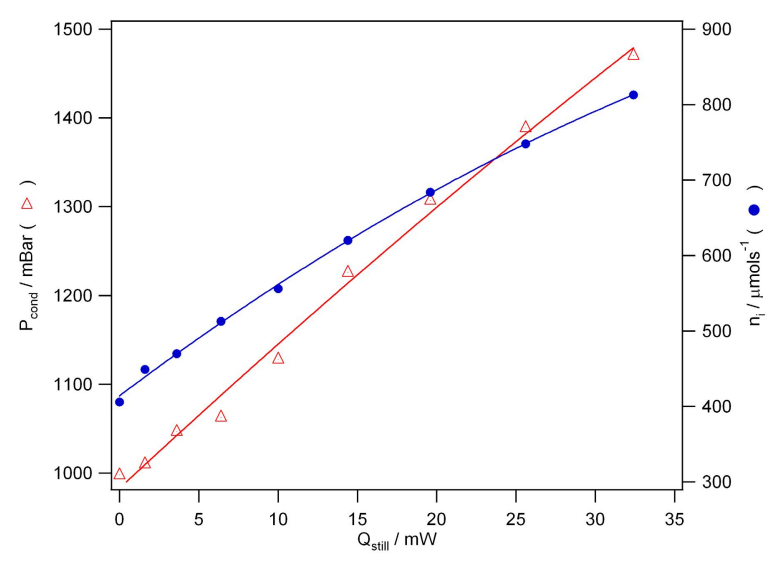

Figure 5. condensing pressure, $P_{\text {cond }}$, and circulation rate, $\dot{n}_{i}$, as functions of still heater power, $\dot{Q}_{s t i l l}$.

\subsection{Baseline performance}

With the DR operating in the test cryostat, the temperature of the second stage of the PTC was measured both with helium circulating, and without. Although the performance of the PTC was not measured independently as part of this work, comparison to the nominal published performance ${ }^{8}$ of the PT410 allows the loading on the PTC to be estimated. With no helium mash in the system, the PTC temperature was measured as $2.91 \mathrm{~K}$. Comparison to the published curves indicate that the background load on the PTC is $\sim 0.2 \mathrm{~W}$. With the DR in normal operation, the PTC second stage temperature was $3.65 \mathrm{~K}$, equivalent to a load of $\sim 0.6 \mathrm{~W}$.

With the DR in continuous operation, measurements were taken of the refrigerator characteristics for different still heater powers, between 0 and $32 \mathrm{~mW}$. Dilution refrigerators are inherently slow systems due to the interaction between the different temperature stages and the large heat capacity of the liquified mixture. After the still heater was set at a current value, the DR was left for several hours to stabilise before values were recorded.

The cooling capacity of the still is depicted in Fig. 4 . The values of $T_{\text {still }}$ were measured using the calibrated Lake Shore RX-102A ruthenium oxide thermometer. For this range of still heater powers, $T_{\text {still }}$ ranged from $910 \mathrm{mK}$ up to $1160 \mathrm{mK}$. This is outside the specification for the still that with $5 \mathrm{~mW}$ load, the still temperature should not exceed $900 \mathrm{mK}$. The consequence of the elevated still temperature is two-fold. Firstly, the temperature of the circulating ${ }^{3} \mathrm{He}$ leaving the still heat exchanger will be higher than optimal, increasing the background loading on the mixing chamber. The elevated temperature will also increase the conduction load onto the mixing chamber. Secondly, the ${ }^{4} \mathrm{He}$ content of the circulating mixture will be increased due to the higher ${ }^{4} \mathrm{He}$ vapour pressure at $1 \mathrm{~K}$ compared to $700 \mathrm{mK}$ (as in a conventional DR). The circulating mixture will still be dominated by ${ }^{3} \mathrm{He}$, but the increase in circulating ${ }^{4} \mathrm{He}$ will have negative effects, such as increasing the load on the PTC first and second stage due to the greater heat capacity of the heavier isotope, and potentially forming bubbles of mixture in the return line and causing alternate heat and cooling of the returning liquid. Operation of the DR in the instrument cryostat with an elevated still temperature will also have further effects on the conductive load to the millikelvin structures and background optical loading on the focal planes.

Conversely, operation of the still at higher temperatures has an advantage in that the vapour pressure (and therefore the circulation rate) of ${ }^{3} \mathrm{He}$ is greatly increased. The increased circulation rate will increase the cooling power of the DR, so long as viscous heating does not become dominant..$^{9}$ Although, as discussed above, a higher still temperature increases the ${ }^{4} \mathrm{He}$ content of the circulating mixture, the the increase in ${ }^{3} \mathrm{He}$ content is also increased (even though the fraction of ${ }^{3} \mathrm{He}$ decreases), increasing the cooling power of the DR. This assumes that the base temperature of the DR is limited by the heat leak, rather than viscous heating effects.

The cause of the poor still performance is likely to be a result of the poor performance of the Joule-Thomson stage. The input temperature of the JT stage is $\sim 4 \mathrm{~K}$ (similar to the PTC temperature), while the gas leaving the $\mathrm{JT}$ is at $\sim 3.5 \mathrm{~K}$, rather than at $1-2 \mathrm{~K}$ as would be desirable following the JT expansion. ${ }^{7} \mathrm{In}$ this DR, the 


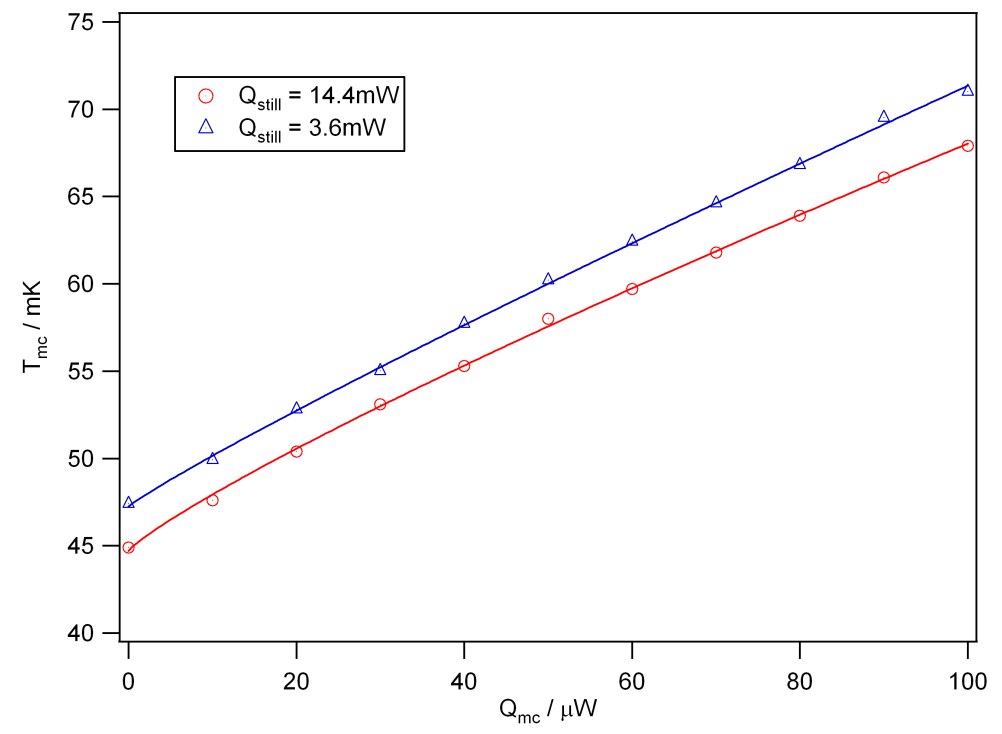

Figure 6. Measured cooling capacity of the mixing chamber, with applied loads of $0-100 \mu \mathrm{W}$. Data is shown for two different values of $\dot{Q}_{\text {still }}$ (and hence two ${ }^{3} \mathrm{He}$ circulation rates).

gas is cooled and condensed in the still heat exchanger, placing additional load on the still since the circulating ${ }^{3} \mathrm{He}$ must be cooled from $3.5 \mathrm{~K}$ to $T_{\text {still }}$ as well as condensed. Taking the heat capacity ${ }^{10}$ of ${ }^{3} \mathrm{He}$ at $\sim 2 \mathrm{~K}$ as $10 \mathrm{~J} / \mathrm{mol}$ and a typical ${ }^{3} \mathrm{He}$ circulation rate of $500 \mu \mathrm{mol} / \mathrm{s}$, the additional load on the still due to the elevated JT temperature is $\sim 10 \mathrm{~mW}$. This is a considerable heat load, given the measured still cooling capacity.

Measurements of the Joule-Thomson stage performance showed that there was little or no temperature change between the input of the heat exchanger and the output of the impedances. The cause of this poor performance may be poor heat exchange with the gas pumped from the still or incorrect impedances on the condensing lines. A possible alternative explanation is a large heat leak onto the JT output. Certainly, the poor JT performance is one of the key limiting factors for this DR.

In addition to measurements of $T_{\text {still }}$ as a function of $\dot{Q}_{\text {still }}$, the condensing pressure $P_{\text {cond }}$ and flow rate, $\dot{n}_{i}$, were recorded. The data is shown in Fig. 5. These measurements were made without the compressor running, and the helium circulating only on the main S3 scroll pump. $P_{\text {cond }}$ varied between 1 and 1.5 bar. The circulation rate varied between 400 and $800 \mu \mathrm{mol} / \mathrm{s}$. The circulation rate data in Fig. 5 is taken from a flow meter in the dilution circuit. The flow meter reading is essentially a mass flow, rather than the flow rate of a specific isotope. The calibration of the flow meter assumes that the circulating gas is pure ${ }^{3} \mathrm{He}$ and ignores the ${ }^{4} \mathrm{He}$ content. If the difference in the ${ }^{3} \mathrm{He}$ and ${ }^{4} \mathrm{He}$ vapour pressures at $1 \mathrm{~K}$ (approximately the still temperature) are considered, $\sim 98 \%$ of the circulating mixture will be ${ }^{3} \mathrm{He}$.

The cooling capacity of the mixing chamber is shown in Fig. 6 for the $\dot{Q}_{m c}$ range $0-100 \mu \mathrm{W}$, for two values of applied still power, $\dot{Q}_{\text {still }}$. As discussed previously, increasing the still temperature (by increasing $\dot{Q}_{\text {still }}$ ) will increase the circulation rate, until viscous heating due to the increased flow outweighs the increased cooling power. Measurements of $T_{m c}$ as a function of $\dot{Q}_{s t i l l}$ for various mixing chamber loads indicated a minimum, occuring at $\dot{Q}_{\text {still }} \sim 14 \mathrm{~mW}$ for low mixing chamber loads, at which point the temperature began to increase due to viscous heating in the mixture or the effect of additional circulating ${ }^{4} \mathrm{He}$. The two capacity curves in Fig. 6 are for the optimum value of $\dot{Q}_{\text {still }}$, and for a lower value.

The requirement that the mixing chamber provide a cooling capacity of $30 \mu \mathrm{W}$ at $65 \mathrm{mK}$ is easily met (with $14 \mathrm{~mW}$ input on the still, the mixing chamber has $>80 \mu \mathrm{W}$ capacity at $65 \mathrm{mK}$ ). The capacity of the mixing chamber at $\sim 120 \mathrm{mK}$ was $400 \mu \mathrm{W}$ compared to a specification of $500 \mu \mathrm{W}$ capacity at that temperature. 
The cooling capacity of a dilution refrigerator, $\dot{Q}_{m c}$, at mixing chamber temperature $T_{m c}$ and with ${ }^{3} \mathrm{He}$ throughput $\dot{n}_{3}$ is given by ${ }^{11}$

$$
\dot{Q}_{m c}=\dot{n_{3}}\left(96 T_{m c}^{2}-12 T_{n}^{2}\right)
$$

where $T_{n}$ is the temperature of the instreaming ${ }^{3} \mathrm{He}$. The total cooling capacity is equal to the applied electrical heat, $\dot{Q}_{\text {Elec }}$, plus the permanently present external heat leak, $\dot{Q}_{\text {Leak }}$, so that

$$
\dot{Q}_{m c}=\dot{Q}_{E l e c}+\dot{Q}_{L e a k}=12 \dot{n}_{3} t
$$

where

$$
t=8 T_{m c}^{2}-T_{n}^{2}
$$

A linear fit to a plot of the applied electrical power, $\dot{Q}_{\text {Elec }}$, as a function of $t$ would allow the ${ }^{3}$ He circulation rate and the magnitude of $\dot{Q}_{\text {Leak }}$ to be recovered from the gradient and intercept of the fitted function. Strictly, in addition to $T_{m c}$, this analysis requires knowledge of the value of $T_{n}$, which is unavailable. However, we know that the limiting case where $\dot{Q}_{m c}=0$ gives the constraint that

$$
T_{m c} \leq T_{n} \leq \sqrt{8} T_{m c}
$$

where the lower limit implies perfect precooling of the instreaming helium to the mixing chamber temperature. The upper limit is the case for no cooling power on the mixing chamber (i.e., the cooling power of the dilution process is balanced exactly by the heat load due to the instreaming ${ }^{3} \mathrm{He}$ ). This assumption is applied to the data plotted in Fig. 7. Although characterisation measurements of the mixing chamber capacity are generally made over the range $0-100 \mu \mathrm{W}$ of applied power, the parameter $t$ is plotted for powers up to $50 \mu \mathrm{W}$ only, since at higher power the measured flow rate was observed to change. This would result in the data deviating from the linear relationship in Eq. (2), since the flow rate is no longer constant. The lines are linear fits to the data; the linear dependance is expected from Eq. (2). For the limit $T_{n}=T_{m c}$, a circulation rate of $(444 \pm 5) \mu \mathrm{Mol} / \mathrm{s}$ and $\dot{Q}_{\text {Leak }}=(75 \pm 1) \mu \mathrm{W}$ are recovered. For the limit $T_{n}=\sqrt{8} T_{m c}$, the same value of $\dot{Q}_{\text {Leak }}$ is found, with $\dot{n}_{3}=(602 \pm 8) \mu \mathrm{Mol} / \mathrm{s}$. The intermediate case, with $T_{n}=2 T_{m c}, \dot{n}_{3}=(518 \pm 6) \mu \mathrm{Mol} / \mathrm{s}$.

From the measured circulation rate as a function of still heater power (Fig. 5), the applied $\dot{Q}_{\text {Still }}$ of $14 \mathrm{~mW}$ gives a flow rate of $620 \mu \mathrm{Mol} / \mathrm{s}$. It is important to note that the measured flow rate will be the total flow of ${ }^{3} \mathrm{He}$ and ${ }^{4} \mathrm{He}$, while the flow rates recovered from the method above will be the flow of ${ }^{3} \mathrm{He}$ only, since the ${ }^{4} \mathrm{He}$ flow contributes nothing to the available cooling power. Allowing for $\sim 2 \%$ of the measured flow to be ${ }^{4} \mathrm{He}$, ${ }^{\mathbb{\pi}}$ the estimated ${ }^{3} \mathrm{He}$ flow rate reduces to $608 \mu \mathrm{Mol} / \mathrm{s}$. This is more consistent with the recovered $\dot{n}_{3}$ value for the case $T_{n}=\sqrt{8} T_{m c}$, at the upper end of the range of values of $T_{n}$. The value for the lower limit of $T_{n}$ implies a $\dot{n}_{3}$ value which is too low in comparison to the measured flow rates. This would imply that the temperature of the instreaming helium is close to the maximum limit of the temperature range, a result of poor heat exchange in the circuit.

The magnitude of $\dot{Q}_{\text {Leak }}$ is very large. However, if we consider the nominal cooling capacity of a conventional MNK126-500 dilution refrigerator as published by Leiden Cryogenics, a heat load of $\sim 75 \mu \mathrm{W}$ equates to a temperature of $\sim 45 \mathrm{mK}$, which is consistent with the measured base temperature of this DR. The implication is that the value of $\dot{Q}_{\text {Leak }}$ is consistent with the measured performance figures. The source of background heat leaks in a DR tend to be conduction loads on to the mixing chamber from shorts to higher temperature stages, poor precooling of the circulating helium and, for a dry dilution refrigerator, vibration from the mechanical cooler. Thermodynamic effects in the helium may also contribute. Given that the recovered circulation rate implies that the temperature of the instreaming helium is close to the upper limit of the temperature range, a major contributor to the heat leak will be poor cooling of the circulating helium in the heat exchangers between

\footnotetext{
${ }^{\text {I }}$ Based on the ratio of ${ }^{3} \mathrm{He}$ and ${ }^{4} \mathrm{He}$ vapour pressure at $\sim 1 \mathrm{~K}$
} 


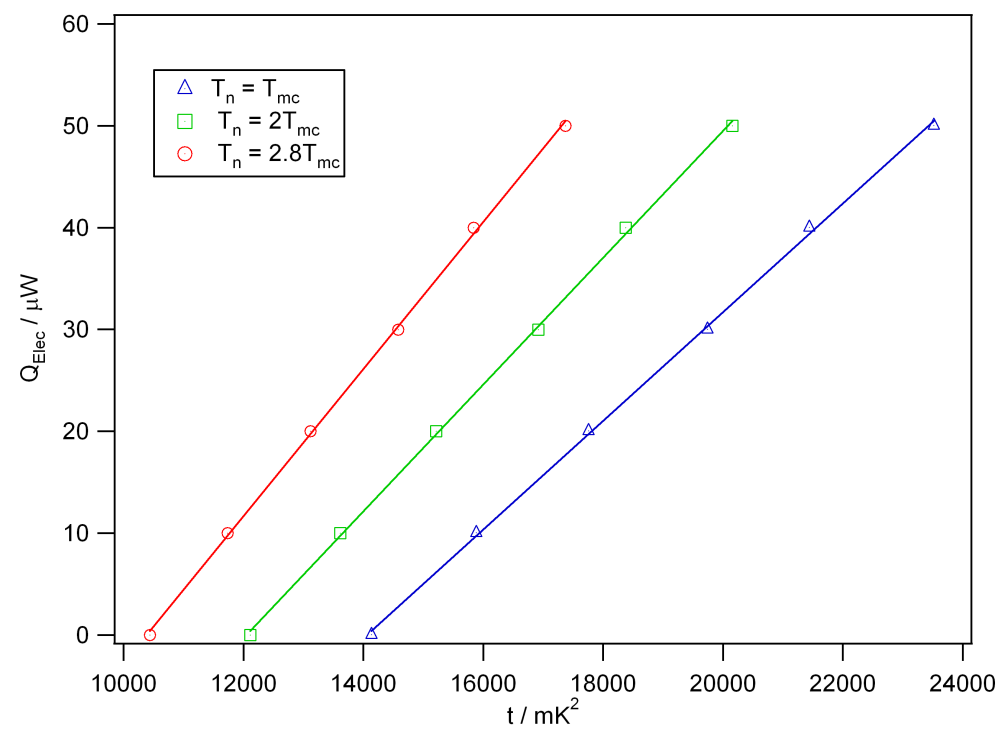

Figure 7. Cooling capacity of the dilution refrigerator as a function of the mixing chamber temperature and the ${ }^{3} \mathrm{He}$ input temperature. For details, see text.

the still and mixing chamber. As discussed previously, although operation of the still at a higher temperature is advantageous in that the circulation rate of ${ }^{3} \mathrm{He}$ is increased, the downside is that the heat leak onto the mixing chamber due to the higher temperature of the instreaming ${ }^{3} \mathrm{He}$ degrades the performance, in addition to the effects of viscous heating.

The data presented and discussed in this section represents the baseline performance for the dilution refrigerator. A key feature of these measurements is the implication that the performance of the DR is dominated by the poor performance of the JT stage, and the apparent large background heat leak on the mixing chamber. The measured still temperatures are higher than would be expected in a conventional dilution refrigerator (generally $\sim 0.7 \mathrm{~K}$ with electrical heating, falling to $\sim 0.4 \mathrm{~K}$ with no applied heat ${ }^{10}$ ). The elevated still temperature would be consistent with greatly increased load due to the cooling and condensation of the relatively warm ${ }^{3} \mathrm{He}$ outflowing from the JT stage. A consequence of the elevated still temperature would be an increased heat leak onto the mixing chamber, as measured from the recovered values of $\dot{Q}_{\text {Leak }}$.

\subsection{Performance in the instrument cryostat}

The important parameters to describe the performance of the DR in the instrument cryostat are the background load on the still and mixing chamber of the system for comparison to the predicted values, and the cooling capacity of the mixing chamber.

With the DR operating in the instrument, it was found that with no additional heating of the still, the still temperature was measured at $1030 \mathrm{mK}$, considerably higher than expected. By comparison to the earlier measurements of the still cooling capacity in Fig. 4, this temperature is consistent with a heat load of $\sim 10 \mathrm{~mW}$, considerably higher than the predicted load of $0.6 \mathrm{~mW}$. The origin of the additional power loading is not clear. The major consequence of the elevated temperature is an increased conduction load on the millikelvin stage of the cryostat.

The typical mixing chamber base temperature in the instrument cryostat was measured as $53 \mathrm{mK}$. The cooling capacity of the mixing chamber is illustrated in Fig. 8, with the baseline cooling capacity in the test cryostat plotted for comparison. The background heat load from the millikelvin system may be determined using the same method as described previously by Eqs. (1-3). The parameter $t$ is plotted against $\dot{Q}_{\text {Elec }}$ up to $30 \mu \mathrm{W}$ in Fig. 9. From a linear fit to the data, a background heatleak of $(87 \pm 2) \mu \mathrm{W}$ is recovered. This is the sum of the 


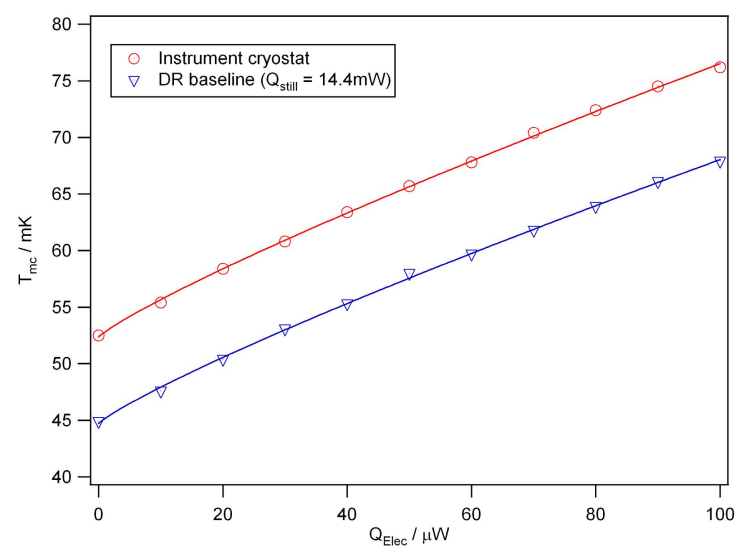

Figure 8. Cooling capacity of the dilution refrigerator in the instrument cryostat.

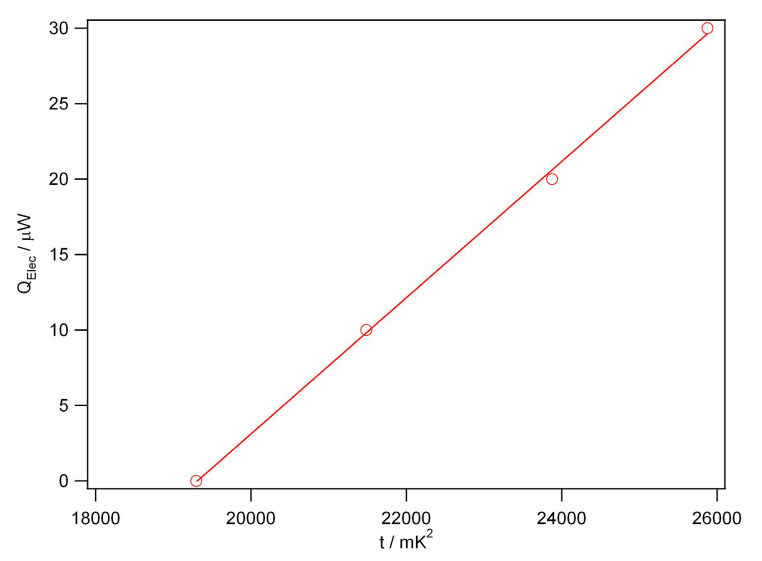

Figure 9. Cooling capacity of the dilution refrigerator as a function of the mixing chamber temperature and the ${ }^{3} \mathrm{He}$ input temperature. For details, see text.

internal heatleak of the dilution refrigerator, determined previously to be $(75 \pm 1) \mu \mathrm{W}$, and the heatload from the millikelvin system. The recovered background power on the mixing chamber is found to be $(12 \pm 2) \mu \mathrm{W}$, which is consistent with the heat load on the millikelvin stage expected from the thermal model. ${ }^{6}$

Measurements of the power dissipation from the detector arrays has determined that each of the 8 detector subarrays dissipates $\sim 5 \mu \mathrm{W}$ at millikelvin temperatures. From the measurements of the DR cooling capacity, the mixing chamber temperature would be expected to increase to $63 \mathrm{mK}$. This value is well within the maximum limit of temperature for the detector arrays to function.

\section{CONCLUSIONS}

The dilution refrigerator is a key sub-system of the SCUBA-2 instrument, and has been found to function close to, although not actually within, the specified performance. With the DR in standalone operation, the typical performance achieved was a base temperature of $44 \mathrm{mK}$, with a measured cooling capacity of $30 \mu \mathrm{W}$ at $53 \mathrm{mK}$ and $100 \mu \mathrm{W}$ at $68 \mathrm{mK}$. This was against a specified performance of $30 \mu \mathrm{W}$ capacity at $65 \mathrm{mK}$ and a goal of $30 \mu \mathrm{W}$ at $35 \mathrm{mK}$. Despite a relatively high base temperature, the DR does meet the cooling capacity specification. The still was found to have a minimum temperature of $0.91 \mathrm{~K}$, with a cooling capacity of $10 \mathrm{~mW}$ at $1.03 \mathrm{~K}$. The specified performance of the still was a temperature of $<0.9 \mathrm{~K}$ with $>5 \mathrm{~mW}$ load. The measured still performance does not meet this specification. The measured temperatures are higher than would be expected for a conventional dilution refrigerator (the still would normally operate at $0.7 \mathrm{~K}$ with electrical heating to maintain the circulation rate ${ }^{10}$ ), although the operating point of the still minimises the mixing chamber temperature due to the balance between the increased circulation rate of ${ }^{3} \mathrm{He}$ at the higher temperature, and the effect of viscous heating and circulation of ${ }^{4} \mathrm{He}$.

More detailed analysis of the mixing chamber performance indicated that the parasitic heat leak was $75 \mu \mathrm{W}$, which is a considerable load. Comparison with the nominal performance of a Leiden Cryogenics DR of this type indicates that the measured elevated base temperature is consistent with a heat load of this level. The origin of this heat leak is believed to be poor precooling of the instreaming ${ }^{3} \mathrm{He}$, which is consistent with the observed elevated temperatures of the still and the Joule-Thomson stage.

With the DR operating in the instrument cryostat, the background loading on the still from the 1-K stage of the instrument was determined to be $\sim 10 \mathrm{~mW}$, considerably higher than expected. The base temperature of the mixing chamber was measured as $53 \mathrm{mK}$, with a background load from the millikelvin stage of (12 \pm 2$) \mu \mathrm{W}$, consistent with the modelled value. With a full complement of detector arrays, dissipating $\sim 40 \mu \mathrm{W}$ of additional power, the mixing chamber temperature is expected to increase to $63 \mathrm{mK}$.

From these measurements, it is concluded that the dilution refrigerator performance is sufficient to allow successful operation of the SCUBA-2 instrument. 


\section{ACKNOWLEDGMENTS}

The authors would like to thank David Gostick, formerly of the UK Astronomy Technology Centre, for input to the early stages of the work described herein.

Thanks are also extended to Ian Bradley of Lancaster University, for a highly useful discussion related to the interpretation of the dilution refrigerator behaviour.

MIH is supported by a Science and Technology Facilities Council studentship.

The SCUBA-2 project is funded by the UK Science and Technology Facilities Council, the JCMT Development Fund and the Canadian Foundation for Innovation.

\section{REFERENCES}

1. W. Holland, M. MacIntosh, A. Fairley, D. Kelly, D. Montgomery, D. Gostick, E. Atad-Ettedgui, M. Ellis, I. Robson, M. Hollister, A. Woodcraft, P. Ade, I. Walker, K. Irwin, G. Hilton, W. Duncan, C. Reintsema, A. Walton, W. Parkes, C. Dunare, M. Fich, J. Kycia, M. Halpern, D. Scott, A. Gibb, J. Molnar, E. Chapin, D. Bintley, S. Craig, T. Chylek, T. Jenness, F. Economou, and G. Davis, "SCUBA-2: a 10,000-pixel submillimeter camera for the James Clerk Maxwell Telescope," in Millimeter and Submillimeter Detectors and Instrumentation for Astronomy III., J. Zmuidzinas, W. S. Holland, S. Withington, and W. D. Duncan, eds., Proceedings of the SPIE 6275, p. 62751E, July 2006.

2. K. Uhlig and W. Hehn, " ${ }^{3} \mathrm{He} /{ }^{4} \mathrm{He}$ dilution refrigerator with Gifford-McMahon precooling," Cryogenics 33, pp. 1028-1031, Nov. 1993.

3. Y. Koike, Y. Morii, T. Igarashi, M. Kubota, Y. Hiresaki, and K. Tanida, "A dilution refrigerator using the pulse tube and GM hybrid cryocooler for neutron scattering," Cryogenics 39, pp. 579-583, Nov. 1999.

4. K. Uhlig, " $3 \mathrm{He} /{ }^{4} \mathrm{He}$ dilution refrigerator with pulse-tube refrigerator precooling," Cryogenics 42, pp. 73-77, Feb. 2002.

5. D. Gostick, D. Montgomery, B. Wall, H. McGregor, M. Cliffe, A. Woodcraft, and F. Gannaway, "The cryomechanical design of SCUBA-2: a wide-field imager for the James Clerk Maxwell Telescope," in Groundbased Instrumentation for Astronomy., A. F. M. Moorwood and I. Masanori, eds., Proceedings of the SPIE 5492, pp. 1743-1754, Sept. 2004.

6. A. L. Woodcraft, F. C. Gannaway, D. C. Gostick, and D. Bintley, "Thermal design of the SCUBA-2 instrument detector stage and enclosure," in Astronomical Structures and Mechanisms Technology., J. Antebi and L. Dietrich, eds., Proceedings of the SPIE 5498, pp. 446-454, Oct. 2004.

7. K. Uhlig, "3 $\mathrm{He} /{ }^{4} \mathrm{He}$ dilution refrigerator without a pumped ${ }^{4} \mathrm{He}$ stage," Cryogenics 27, pp. 454-457, Aug. 1987.

8. C. Wang and P. E. Gifford, "Development of $4 \mathrm{~K}$ pulse tube cryorefrigerators at cryomech," in Advances in Cryogenic Engineering CEC, S. Breon, M. Dipirro, D. Glaister, J. Hull, P. Kittel, V. R. R. Pecharsky, J. Theilacker, S. van Sciver, J. I. Weisend, and A. Zeller, eds., American Institute of Physics Conference Series 613, pp. 641-648, May 2002.

9. D. I. Bradley. Personal communication.

10. F. Pobell, Matter and Methods at Low Temperatures, Springer-Verlag, third ed., 2007.

11. O. V. Lounasmaa, Experimental Principles and Methods Below 1K, Academic Press, 1974. 